\title{
Optical properties of intermetallic compounds from first principles calculations: a search for the ideal plasmonic material
}

\author{
M G Blaber, M D Arnold and M J Ford \\ Institute for Nanoscale Technology, Physics and Advanced Materials, University of \\ Technology Sydney, PO Box 123, Broadway 2007, NSW, Australia \\ Email: mike.ford@uts.edu.au
}

PACS: 71.15.Mb, 71.20.Lp, 71.45.Gm, 77.22.Ch, 77.22.Gm, 78.20.Bh, 78.20.Ci

\begin{abstract}
First principles calculations have been used to predict the optical properties for a range of intermetallic compounds for which little or no experimental optical data is currently available. Density Functional Theory (DFT) combined with the Random Phase Approximation (RPA) is used to calculate the dielectric functions for these compounds. The aim of this work is to investigate how the band-edge and plasma frequency vary with composition in order to identify materials with promising plasmonic properties. Towards this end the intermetallic compounds chosen are composed of elements, which on their own, have reasonable optical properties for plasmonic applications. The relative position of the band edge to the plasma frequency is most favourable in the simple binary compounds formed from the alkali plus noble metals $\mathrm{NaAu}, \mathrm{KAu}$ and $\mathrm{KAg}$. In particular, for KAu the band edge and plasma frequency occur almost at the same frequency, and hence the imaginary part of the dielectric function is practically zero for frequencies below the plasma frequency. In addition, the plasma frequency in this compound is at relatively low frequency promising a material with strong plasmon response in the infra-red.
\end{abstract}

\section{Introduction}

The free-electron character of metals has a profound effect on their optical properties, and has been exploited, since ancient times, for applications such as mirrors and stained-glass. The previous century has seen steady progress in our understanding of the underlying physics of free-electron metals. More recently, plasmonics has become a very active research area in its own regard driven by theoretical and experimental developments and the potential technological applications of plasmonic devices. New applications have appeared in many areas including medical diagnostics and therapeutics [1-3], subwavelength colour imaging [4], communications and computing [5-7], to name but a few.

The limiting factor for many applications is optical loss, generally due to interband transitions. This is empirically described through the imaginary part of the refractive index $m$, or equivalently through the imaginary part of the permittivity $\varepsilon^{\prime \prime}$. Considerable effort in plasmonics research is directed towards designing structures that mitigate this loss, often by tuning operating frequencies to minimise imaginary permittivity. While some useful gains have been achieved, optical performance is often still far from optimal because the loss for usable metals at the desired operating frequency is simply too high. A good example of this is the superlens [8]. Here, periodic arrays of metallic resonators give a negative refractive index 'meta-material' $[9,10]$. Superlenses operating at microwave wavelengths have been demonstrated [11], however, achieving similar performance at optical wavelengths has been elusive. This is due to the poor performance of metals in the visible region, and to the increased difficulty of constructing nanoscale resonators [12-14]. Considerable effort has been aimed at designing optimal structures for optical meta- 
materials, the most promising structures appear to be the fishnet structures [15, 16]. More recently negative refraction has been reported using nanowires [17].

A partial superlens can easily be constructed using a homogeneous layer of metal, for example silver, but again performance is degraded by metal losses [18]. The group I metals are the closest to freeelectron like, and indeed potassium has very promising optical properties for plasmonic applications, as we have already demonstrated [19]. However, they are also chemically very reactive and therefore rather limited from a practical perspective. Although, we should point out that potassium nanoparticles have been made and their optical properties measured [20,21].

The optical performance of a metal for many plasmonic applications will be dictated by the relative positions of the plasma frequency and inter-band transitions. For example, in gold at visible wavelengths the plasmonic response is significantly modified by the presence of transitions. Clearly, there is little scope to ameliorate the situation using elemental metals, however with inter-metallic compounds and alloys it should be possible to band engineer the material in such a way as to optimise the plasmonic response. This is the central challenge in the present work.In light of the optical properties of the Noble, Alkali and Group III metals, here we shall calculate, from first principles, the optical properties of a series of Alkali (A), Noble (N) and Group III (G) metal compounds with a particular focus on the Alkali-Noble binary compounds. To our knowledge, no metal alloy or compound has been specifically presented as a candidate for plasmonics, although some have shown promise [22]. While the optical spectra of some alloys have been measured or calculated, this has generally been aimed at understanding electronic states close to the Fermi energy [23] rather than systematically investigating interesting optical properties [24].

The Alkali Noble compounds have received attention due to the semiconducting nature of the CsAu compound (band gap 2.86eV) [25, 26]. The metal-semiconductor transition between KAu and RbAu has been attributed to increasing electropositivity of alkali elements down the periodic table [27]. The LiAu, $\mathrm{CsAu}$ and $\mathrm{RbAu}$ compounds can crystallise in the simple cubic $\mathrm{CsCl}$ structure. The lack of experimental evidence supporting the crystallization of $\mathrm{NaAu}$ and $\mathrm{KAu}$ in the same structure has been ascribed to insufficient interstitial screening of the noble atoms by their alkali counterparts [27]. The most promising of the compounds studied previously is $\mathrm{Li}_{2} \mathrm{AgIn}$. Zwilling et al [22] report interesting results for this compound, the most surprising of which is that the imaginary part of the permittivity is zero at $2 \mathrm{eV}$.

\section{Method}

We use the SIESTA methodology to perform our electronic structure calculations [28, 29]. SIESTA is an implementation of Density Functional Theory using the linear combination of atomic orbitals approximation and periodic boundary conditions. Core electrons are described by non-local normconserving Troullier-Martins type pseudopotentials [30]. Relativistic core corrections are used for elements above Gallium. Valence electrons are described using numerical atom-centred basis sets with orbitals confined such that the energy increase due to the confinement is no more than $5 \mathrm{mRy}$ [31]. A 350 Ry effective real space integration grid cut-off is employed and a $25 \mathrm{meV}$ Fermi smearing is used to assist convergence. We use the exchange correlation functional by Perdew, Burke and Ernzerhof [32] and a Brillouin integration grid built using the Monkhorst-Pack [33] scheme with 29x29x29 points for the self consistent calculation. Full geometry optimisations were performed on all structures and an optical Brillouin zone integration grid of $65 \times 65 \times 65$ was used, resulting in approximately 20000 points in the irreducible Brillouin zone. Although the optical spectra are well converged before this point, the plasma frequency $\omega_{\mathrm{p}}$ is only converged to within $2 \%$. First order time dependent perturbation theory is used to calculate the interband component of the imaginary part of the permittivity:

$$
\varepsilon^{\prime \prime}(\omega)=\frac{8 \pi^{2}}{3 V} \sum_{i, j} \int_{B Z} d \mathbf{k} \frac{2 f_{\mathbf{k}, i}\left(1-f_{\mathbf{k}, j}\right)\left|\mathbf{P}_{i, j}\right|^{2}}{\left(E_{\mathbf{k}, j}-E_{\mathbf{k}, i}\right)\left[\left(E_{\mathbf{k}, j}-E_{\mathbf{k}, i}\right)^{2}-\omega^{2}-\mathrm{i} \delta\right]},
$$

where $V$ is the unit cell volume, $\mathbf{P}_{i, j}$ are the momentum matrix elements between initial band $i$ and final band $j$ including a correction for the use of non-local pseudopotentials [34], $f$ is the Fermi-Dirac distribution function. A comparison with experimental data shows a consistent overestimation of the momentum matrix elements, yielding spectra that show an overdamping of plasmon resonances. Although the real part of the permittivity plays an essential role in the applicability of intermetallics to 
plasmonics, as it determines not only the location of the resonance but also it's dispersion characteristics, in the interest of brevity we shall only discuss the location of the plasma frequency as calculated by the Random Phase Approximation (RPA):

$$
\omega_{p}^{2}=\frac{8 \pi}{3 V} \sum_{i, \mathbf{k}}\left|\frac{d E_{\mathbf{k}, i}}{d \mathbf{k}}\right|^{2} \delta\left(E_{\mathbf{k}, i}-E_{F}\right),
$$

The real part of the permittivity can be calculated from the Kramers-Kronig relation [35], although obtaining a reliable value requires integrating the imaginary component over a very wide wavelength region. A number of more sophisticated approaches, such as time-dependent DFT [29] are now available for calculating optical properties, but are limited in applicability and would struggle with the relatively large unit cell sizes in some of our calculations. Moreover, we are interested in identifying trends in the optical properties across a series of intermetallic compounds and hence the RPA, even with its known shortcomings, is a suitable starting point.

\section{Optical Properties of Intermetallic Compounds}

\subsection{Noble-Group III Compounds}

The Noble Group III compounds take on the fluorite structure (space group Fm-3m) with the Noble metal on the standard FCC sites and the Group III atoms form a simple cubic structure in the centre of the FCC cell.

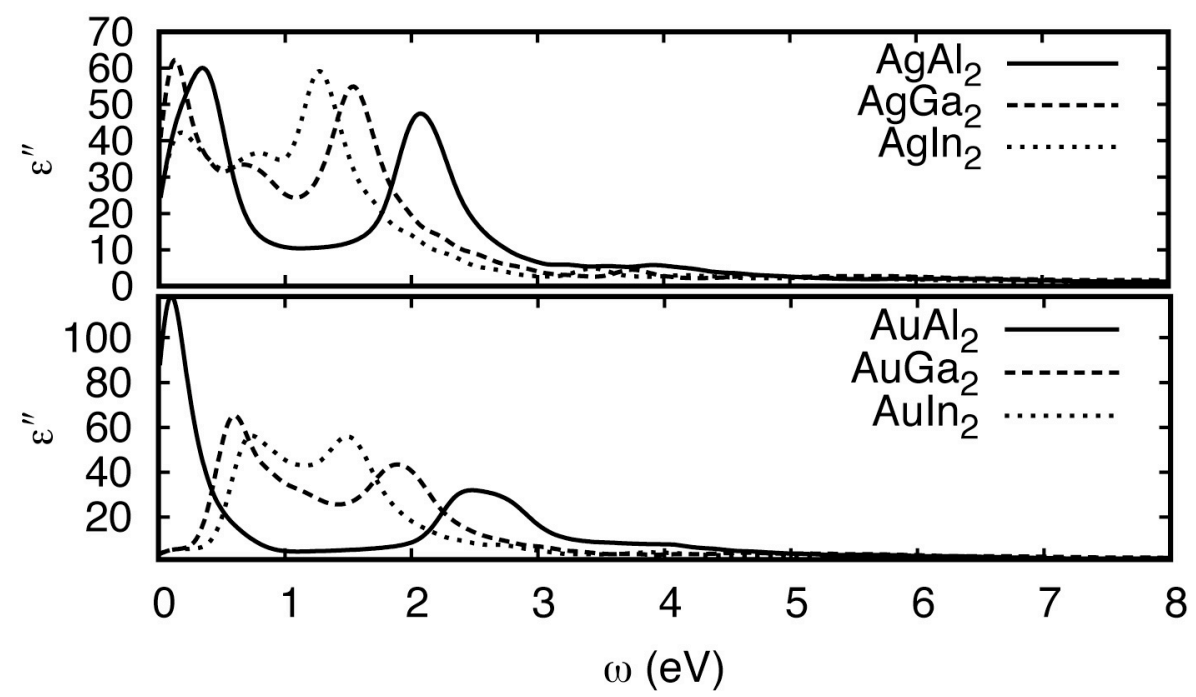

Figure 1. Interband transition spectra for various Noble-Group III Compounds. Due to uniform broadening over the entire spectrum the transition probability at zero energy is positive.

Figure 1 shows the calculated interband contribution to the imaginary part of the permittivity. $\mathrm{AuAl}_{2}$ has two peaks in the imaginary part of the permittivity with the second set of transitions centered at about $2.4 \mathrm{eV}$. The secondary band edge (SBE) for these transitions is at about $2.2 \mathrm{eV}$. It would seem natural to associate this feature with the band-edge (BE) in the spectrum of elemental gold at about $2.5 \mathrm{eV}$. However, the Au d-bands are too far below the Fermi energy to contribute to the spectra [36], also the Secondary Band Edge (SBE) in $\mathrm{AgAl}_{2}(2.2 \mathrm{eV})$ is shifted from the band edge (BE) of silver at $3.9 \mathrm{eV}$ [37]. See table 1. Comparison of the calculated and experimental values of SBE and plasma frequency for the Au compounds in table 1 demonstrates that the calculations are relatively good at reproducing the optical properties.

The minimum transition strength in the visible to near-infrared for $\mathrm{AuAl}_{2}$ is 4.6 at approximately 1.1 $\mathrm{eV}$, however it does drop as low as 1.4 in the ultraviolet, at $7.2 \mathrm{eV}$, just $1 \mathrm{eV}$ below the plasma frequency. As the lattice constant is increased from $\mathrm{Al}$ to $\mathrm{In}$, the $\mathrm{BE}$ of the $\mathrm{Au}$ compounds steadily increases from approximately $0 \mathrm{eV}$ up to $0.6 \mathrm{eV}$ for $\mathrm{AuIn}_{2}$ while the $\mathrm{SBE}$ continually decreases from $2.2 \mathrm{eV}$ in $\mathrm{AuAl}_{2}$ to $1.35 \mathrm{eV}$ in $\mathrm{AuIn}_{2}$, bringing with it the possibility for plasmonic activity above $2 \mathrm{eV}$. Unfortunately $\varepsilon^{\prime \prime}$ 
decreases only to a value of 1.3 at the plasma frequency, $\omega_{p}(7.21 \mathrm{eV})$ resulting in a poor plasmon response across all of the visible and near UV.

In contrast to $\mathrm{Au}$, the $\mathrm{BE}$ for the $\mathrm{Ag}$ compounds moves to lower energy from $\mathrm{Al}$ to $\mathrm{Ga}$, and then shifts slightly back towards higher energies for In. The plasma frequencies for the Ag - Group III compounds are $7.78,8.06$ and 7.62 for $\mathrm{Al}, \mathrm{Ga}$ and In respectively. Again, the lowest value of $\varepsilon^{\prime \prime}$ is for the indium intermetallic compound with a value of 0.89 at $7.62 \mathrm{eV}$. Neither $\mathrm{AgAl}_{2}$ or $\mathrm{AgGa}_{2}$ have imaginary permittivity less than 1 at any energy lower than the plasma frequency.

Table 1..Plasma frequency and Secondary Band Edge of Noble-Group III compounds.

\begin{tabular}{ccccc}
\hline & \multicolumn{2}{c}{ Calculated } & \multicolumn{2}{c}{ Experimental [38] } \\
\cline { 5 - 5 } $\begin{array}{c}\text { Comp } \\
\text { ound }\end{array}$ & SBE (eV) & $\omega_{\mathrm{p}}(\mathrm{eV})$ & SBE $(\mathrm{eV})$ & $\omega_{\mathrm{p}}(\mathrm{eV})$ \\
\hline $\mathrm{AuAl}$ & & & & \\
$\mathrm{AuGa}_{2}$ & 1.2 & 8.25 & 2.2 & 6.7 \\
$\mathrm{AuIn}_{2}$ & 1.3 & 7.34 & 2.0 & 7.3 \\
$\mathrm{AgAl}_{2}$ & 1.9 & 7.78 & 1.7 & 7.2 \\
$\mathrm{AgGa}_{2}$ & 1.4 & 8.06 & & \\
$\mathrm{AgIn}_{2}$ & 1.2 & 7.62 & & \\
\hline
\end{tabular}

\subsection{Alkali-Noble-Group III Compounds}

The optical properties of $\mathrm{Li}_{2} \mathrm{AgIn}$ as measured by Zwilling, Schmidt and Weiss in reference [22] show some incredible promise. The imaginary part of the permittivity goes to zero at $2 \mathrm{eV}$. Unfortunately, in the same work they report the $\mathrm{LiCd}_{2} \mathrm{In}$ compound as having an imaginary permittivity slightly less than zero $(-2$ at $3.1 \mathrm{eV})$. It is possible that surface defects or Kramers-Kronig analysis caused the anomalous values for $\varepsilon^{\prime \prime}$. It crystallizes in the Heussler Type Fm-3m structure [22] with 16 atoms per unit cell. The structure can be described as a group of 8 BCC cells making up a cube. The corner atoms of each BCC cell are composed of alternating lithium and indium atoms and the centers of alternating BCC cells are lithium and silver atoms.

Here, we have calculated compounds between $\mathrm{Li}_{2} \mathrm{AgAl}$ and $\mathrm{K}_{2} \mathrm{AuIn}$ and also compounds from $\mathrm{LiAg}_{2} \mathrm{Al}$ to $\mathrm{KAu}_{2} \mathrm{In}$. We will use the notation noble 2, or N2 to denote the latter group, and alkali 2 or A2 to denote the former. All these compounds have the same Fm-3m space group [39]. Figure 2 shows a map of the interband component of $\varepsilon^{\prime \prime}$ for all the $\mathrm{A} 2$ compounds. The SBE for the $\mathrm{Li}_{2}$ compounds follows the same trend as the plasma frequency. $\mathrm{Li}_{2} \mathrm{AgAl}$ has the lowest absorption with $\varepsilon^{\prime \prime}=0.9$ at $2.5 \mathrm{eV}$. Only the $\mathrm{Li}_{2}$ compounds have $\varepsilon^{\prime \prime}<2$ at any energy below, but reasonably close to the plasma frequency. $\mathrm{The} \mathrm{Na}_{2}$ compounds meet this criteria at the plasma frequency and the $\mathrm{K}_{2}$ compounds only have $\varepsilon^{\prime \prime}<2$ at energies higher than $\omega_{\mathrm{p}}$. $\mathrm{Na}_{2} \mathrm{AgAl}$ has the largest transition at $0.8 \mathrm{eV}\left(\varepsilon^{\prime \prime}=51.8\right)$.

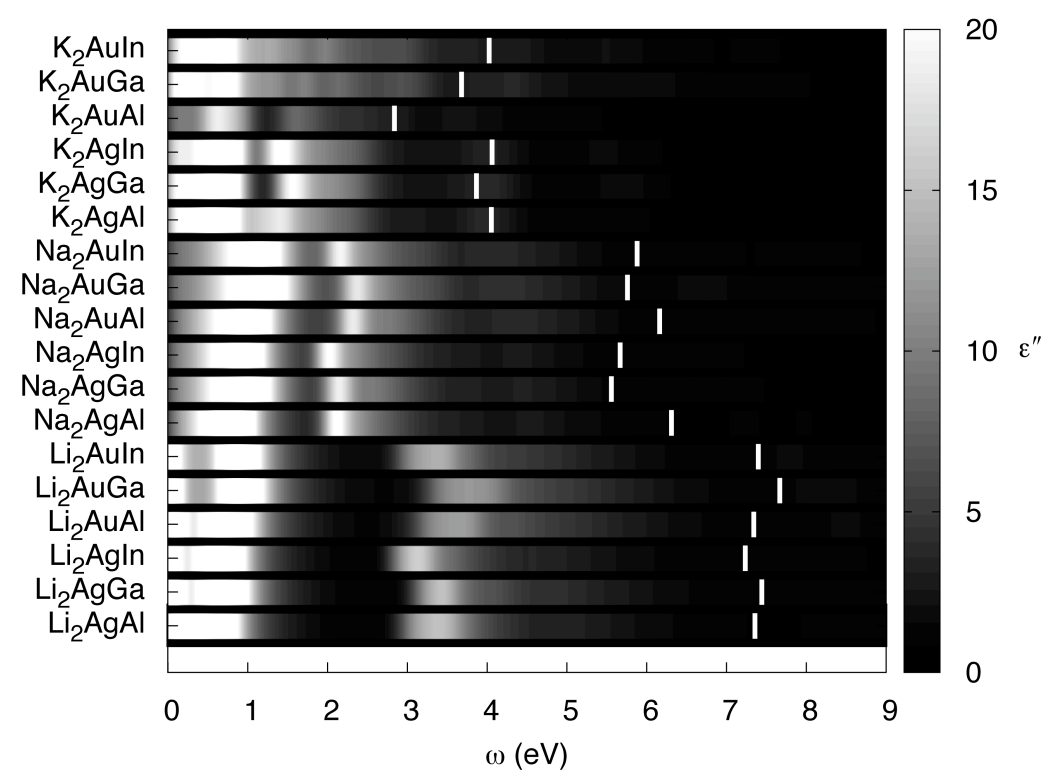


Figure 2. Interband transition spectra of all the A2 compounds. White bars indicate the position of the plasma frequency. The magnitude of $\varepsilon^{\prime \prime}$ has been truncated to 20 to aid visibility.

Interband transition spectra for the N2 compounds are shown in figure 3. Almost half of these compounds have transitions with magnitude of $\varepsilon^{\prime \prime}$ greater than 50 at energies lower than $2 \mathrm{eV}$. $\operatorname{LiAg}_{2} \mathrm{Ga}$ has both the highest value of $\varepsilon^{\prime \prime}, 140$ at an energy below $0.1 \mathrm{eV}$ and second lowest $\varepsilon^{\prime \prime}, 1.5$ at an energy of $2.0 \mathrm{eV} . \mathrm{KAg}_{2} \mathrm{Al}$ has the lowest imaginary permittivity at $3.0 \mathrm{eV}$ with a value of 1.3 . The $\mathrm{LiAg}_{2}$ and $\mathrm{NaAg}_{2}$ compounds have permittivity between 1.2 and 1.5 at a maximum of $2 \mathrm{eV}$ below their plasma frequencies which sit in the $7.5-8.5 \mathrm{eV}$ region.

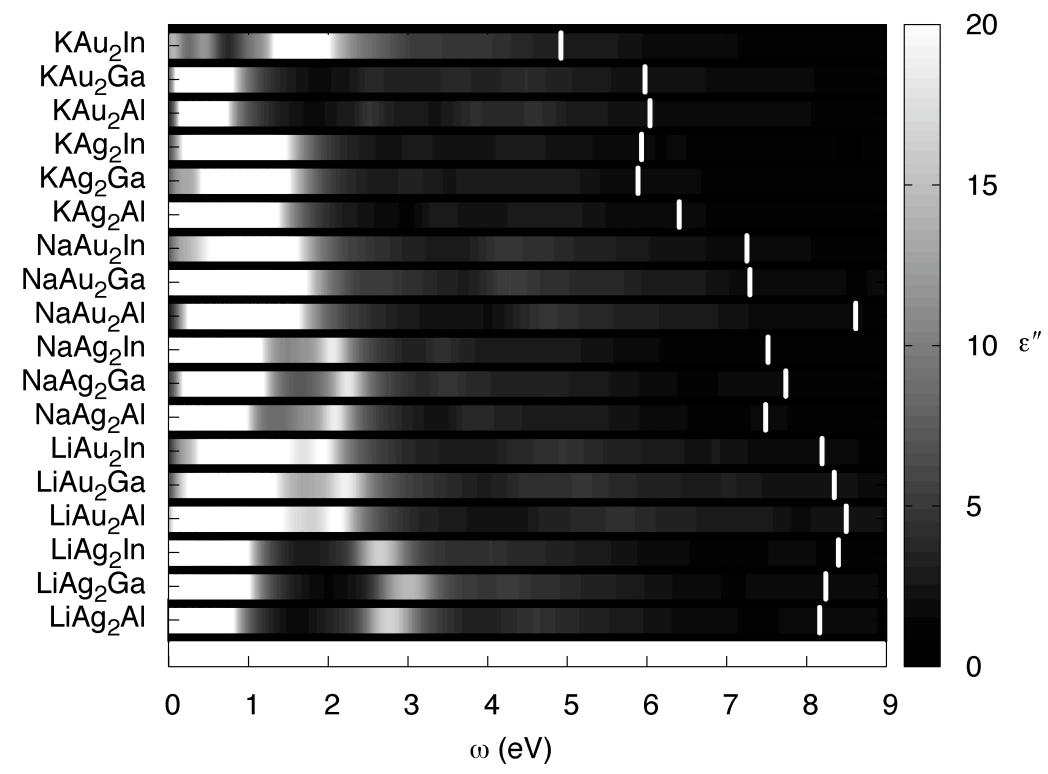

Figure 3. Interband transition spectra of all the Alkali N2 Group III compounds. White bars indicate the position of the plasma frequency. The magnitude of $\varepsilon^{\prime \prime}$ has been truncated to 20 to aid visibility.

For both the N2 and A2 compounds we can see from Figures 2 and 3 that the plasma frequency decreases on descending the alkali group. This would also follow from the plasma frequency calculated from the Drude model

$$
\omega_{p}=\sqrt{\frac{n_{e} e^{2}}{m_{e} \varepsilon_{0}}},
$$

where $n_{e}$ is the free electron density, $e$ is the electron charge, $m_{e}$ the electron mass, and $\varepsilon_{0}$ is the permittivity of free space. If we assume that the compounds donate their valence electrons to the electron gas then the electron density in equation 3 is inversely proportion to the unit cell volume or lattice constant cubed. Hence the Drude plasma frequency is inversely proportional to the lattice constant to the power 3/2. We have compared Drude plasma frequencies with our RPA calculated values in Figure 4. There is a reasonable straight line correlation between the two data sets, the Drude plasma frequency, however, falls consistently below the RPA value by up to a factor of 2 in the case of $\mathrm{K}_{2}$ compounds. These compounds clearly donate fewer valence electrons to the free-electron gas than any of the other compounds. Lattice constants for both the $\mathrm{Li}$ and $\mathrm{Na}$ compounds remain almost constant, however, the $\mathrm{K}_{2}$ compounds have a lower lattice constant than their $\mathrm{KN}_{2}$ counterparts. 


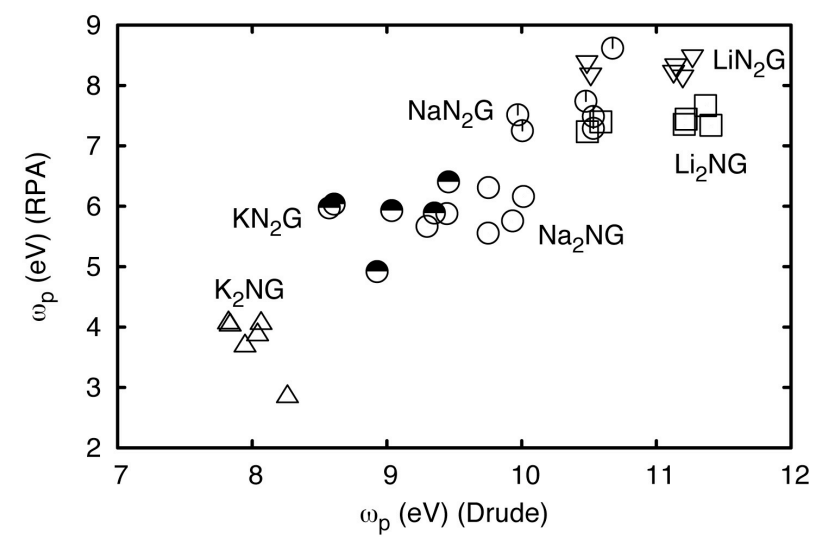

Figure 4. Comparison of the plasma frequency as calculated by the Random Phase Approximation (RPA) and that calculated by the standard Drude free electron gas formalism. N=Noble, G=Group III.

\subsection{Alkali-Noble Compounds}

Of all the compounds studied here, the Alkali-Noble intermetallic compounds are the most interesting, mainly due to the increasing energy of the band edge as the period is increased for both the Alkali and Noble metals. Figure 5 shows the interband transition spectra of the Alkali Noble compounds. Although the band edges (see table 3) of these compounds are much lower than Ag and Au, which have band edges of $3.9 \mathrm{eV}$ and $2.25 \mathrm{eV}$ respectively [37], they are significantly higher than any of the Alkali Noble Group III and Noble Group III compounds. The onset of interband transitions increases in energy for substitutions of the alkali metal atom down the period for both silver and gold compounds. Conversely, the plasma frequency decreases down the period as the lattice constant increases, indicating that there should be some crossover point.

In order to relate optical properties to the band structure and hence attempt to engineer the optical properties we need to understand the transition characteristics and the relative contributions transitions make to the complex permittivity. We employ a variant of the conventional 'fat-band' analysis (see for example [40]) where instead of projecting band character onto a band structure, we sum all transitions originating from (or ending at) a single k-point in the band and then denote the magnitude of this sum by a circle drawn on the band structure. That is, we draw circles on the band structure given by:

$$
\begin{aligned}
& r_{i}=\alpha \sum_{\partial \mathbf{k}<\sigma} \sum_{j} \frac{8 w \pi^{2}}{3 V} \frac{2 f_{\mathbf{k}, i}\left(1-f_{\mathbf{k}, j}\right)\left|\mathbf{P}_{i, j}\right|^{2}}{\left(E_{\mathbf{k}, j}-E_{\mathbf{k}, i}\right)} \\
& r_{j}=\alpha \sum_{\partial \mathbf{k}<\sigma} \sum_{i} \frac{8 w \pi^{2}}{3 V} \frac{2 f_{\mathbf{k}, i}\left(1-f_{\mathbf{k}, j}\right)\left|\mathbf{P}_{i, j}\right|^{2}}{\left(E_{\mathbf{k}, j}-E_{\mathbf{k}, i}\right)}
\end{aligned}
$$

where $r$ represents the radius of the circle on the band diagram and $\alpha$ is a normalizing constant. Subscripts $i$ and $j$ denote initial and final states for the transition. We then sum the magnitude of transitions over $\mathrm{k}$ points in the optical BZ that occur within a distance $\sigma$ of the k-point of interest on the band structure. The results of this analysis can be seen in figures 7 and 8 . 


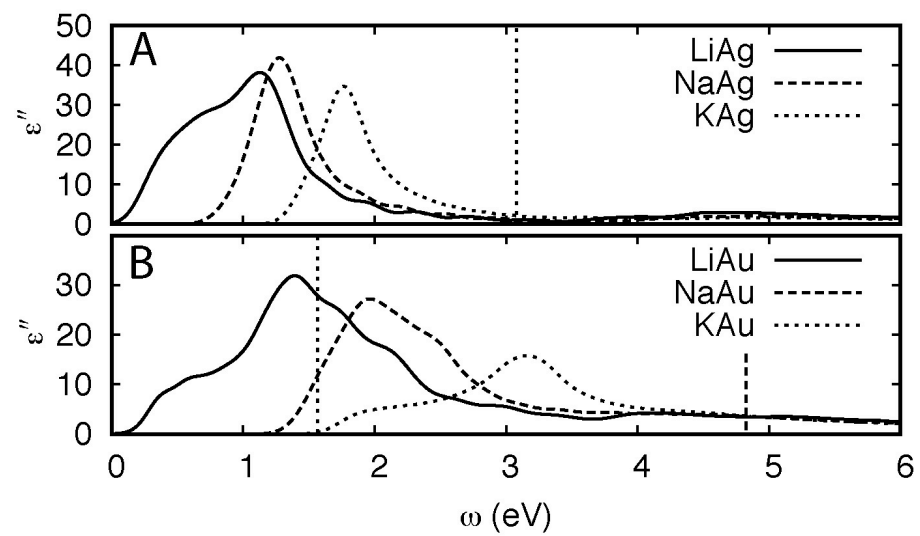

Figure 5. .Optical properties of A) the Alkali Ag and B) the Alkali Au compounds. Vertical lines represent the position of the plasma frequency for those compounds with $\omega_{\mathrm{p}}<6.0 \mathrm{eV}$

Table 3. Band edges for selected metals and compounds. Elemental band edges taken from [37].

\begin{tabular}{rcrc}
\hline Metal & Band Edge $(\mathrm{eV})$ & Metal & Band Edge $(\mathrm{eV})$ \\
\hline $\mathrm{Ag}$ & 3.9 & $\mathrm{Au}$ & 2.25 \\
$\mathrm{LiAg}$ & 0.25 & $\mathrm{LiAu}$ & 0.3 \\
$\mathrm{NaAg}$ & 0.95 & $\mathrm{NaAu}$ & 1.58 \\
$\mathrm{KAg}$ & 1.55 & $\mathrm{KAu}$ & 1.72 \\
\hline
\end{tabular}

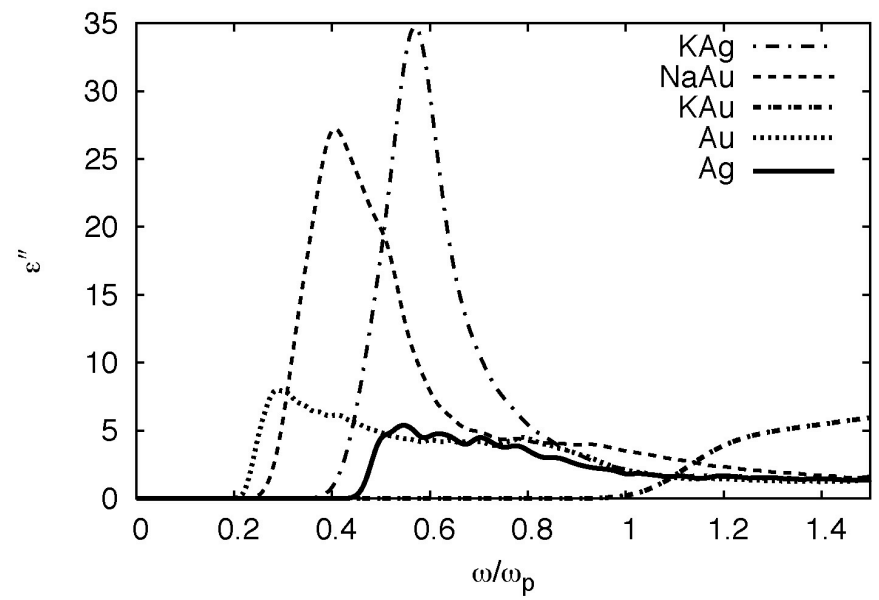

Figure 6. Absorption in Alkali Noble compounds with Band Edge to plasma frequency ratio better than Gold.

$\mathrm{KAg}, \mathrm{NaAu}$ and $\mathrm{KAu}$ all have plasma frequencies less than $6 \mathrm{eV}$ causing the ratio of the position of the band edge to the plasma frequency to be higher than that of elemental Gold. This makes these materials particularly interesting in the infrared. Most notably, KAu has almost no interband transitions below the plasma frequency. Figure 6 shows the relationship between the plasma frequency and the band edge for compounds which have a band edge to plasma frequency ratio of less than 0.2. Both Gold and Silver are good plasmonic materials in part due to this ratio. Any resonance that occurs below the band edge is damped solely by the intraband component of the permittivity. Moreover, as the absolute value for $\mathrm{KAu}$ is so low, the contribution of Drude like damping to the intraband component of the permittivity will be negligible down to very low energies. For example, considering just the plasma frequency for KAu at $1.54 \mathrm{eV}$, we can calculate the position of the sphere resonance to be at approximately $1390 \mathrm{~nm}$.

The nature of the interband spectra in figure 5 becomes more obvious from figures 7 and 8 . The radius of the circles in figures 7 and 8 denote the strength of all transitions originating (or ending) at that particular point in the band structure. Transitions near the special point $\mathrm{M}$ in figure 7 for $\mathrm{LiAg}$ and figure 8 for LiAu are responsible for the low energy structure in $\varepsilon^{\prime \prime}$. As the lattice constant is increased by substituting larger radii alkali metal atoms, bands just below the Fermi energy at $\mathrm{X}$ move from doubly degenerate at $\mathrm{M}$ to well separated and transitions become allowed. In $\mathrm{NaAu}$ this results in the $2 \mathrm{eV}$ peak in the spectrum. That particular transition is still forbidden in NaAg however, and the spectrum is missing 
that characteristic peak. In certain places on the band diagrams (figure $8 \mathrm{NaAu}$ at $\mathrm{M}$ ) double degeneracy of bands above the Fermi energy gives the appearance of missing high energy transitions, however, there are in fact twice as many circles. The small $2 \mathrm{eV}$ peak in KAu is caused by a small number of large magnitude transitions around $\mathrm{X}$, and there is almost a continuous increase in energy for transitions from $\mathrm{X}$ to $\mathrm{M}$ where a large number of small magnitude transitions are responsible for the $3.2 \mathrm{eV}$ peak. Bands above and below the Fermi energy in KAg are separated by $1.75 \mathrm{eV}$ across the entire surface of the BZ.

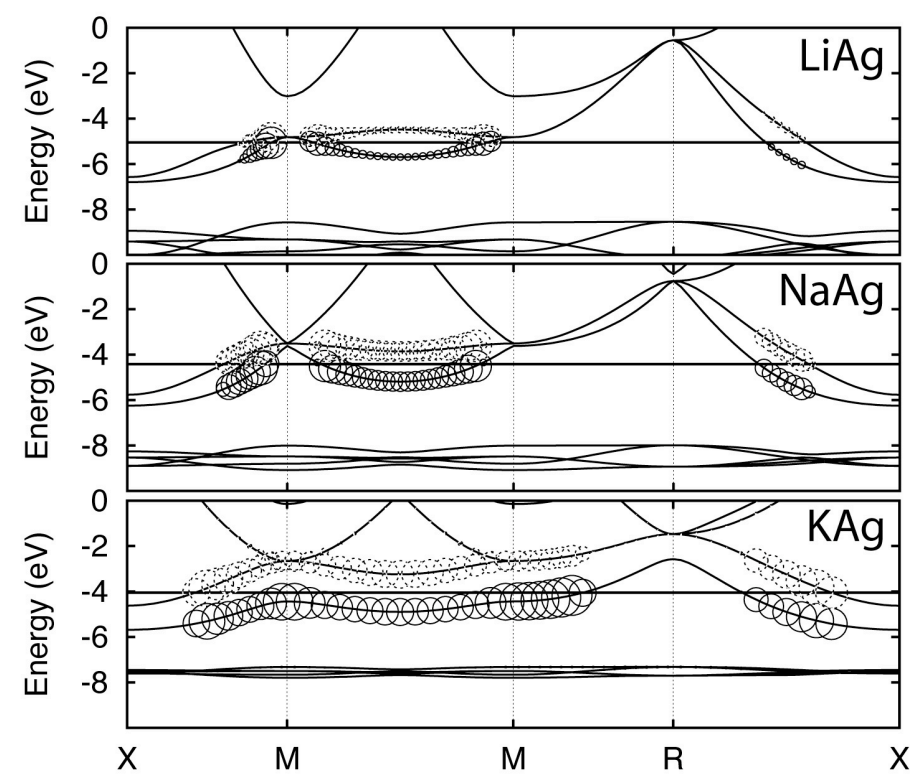

Figure 7. (Colour Online) Band diagram for Alkali Ag compounds. Included is the sum of transitions from a band below the Fermi energy (solid circles) to all bands above, and the sum of transitions to a band above the Fermi energy (broken circles) from all bands below. The line M-M intersects X-R, see figure 8 .

Table 4. Plasma frequencies of Alkali Noble compounds.

\begin{tabular}{rrrc}
\hline Metal & $\omega_{\mathrm{p}}(\mathrm{eV})$ & Metal & $\omega_{\mathrm{p}}(\mathrm{eV})$ \\
\hline $\mathrm{LiAg}$ & 7.28 & $\mathrm{LiAu}$ & 7.2 \\
$\mathrm{NaAg}$ & 6.47 & $\mathrm{NaAu}$ & 4.84 \\
$\mathrm{KAg}$ & 3.10 & $\mathrm{KAu}$ & 1.54 \\
\hline
\end{tabular}

We see a reduction in the plasma frequency due to the gradient of the bands as well as a splitting of the bands in both the Ag and Au Alkali compounds characteristic of increasing ionic bond character in line with the observations of Koenig et al [26] and Grosch et al [27].

A more complete way to view the nature of the transitions is to plot the transition density for all kpoints in the Brillouin zone. Figure 8 shows the density of transitions in the upper half of the Brillouin zone for KAg. Note the transparency of sections around X and R corresponding to (almost completely) forbidden transitions. The centre of the structure is essentially hollow, however there is enough density, that the sum contributes to the background transitions (i.e. above $\sim 4 \mathrm{eV}$ ) in the spectra of the AlkaliNoble compounds. 


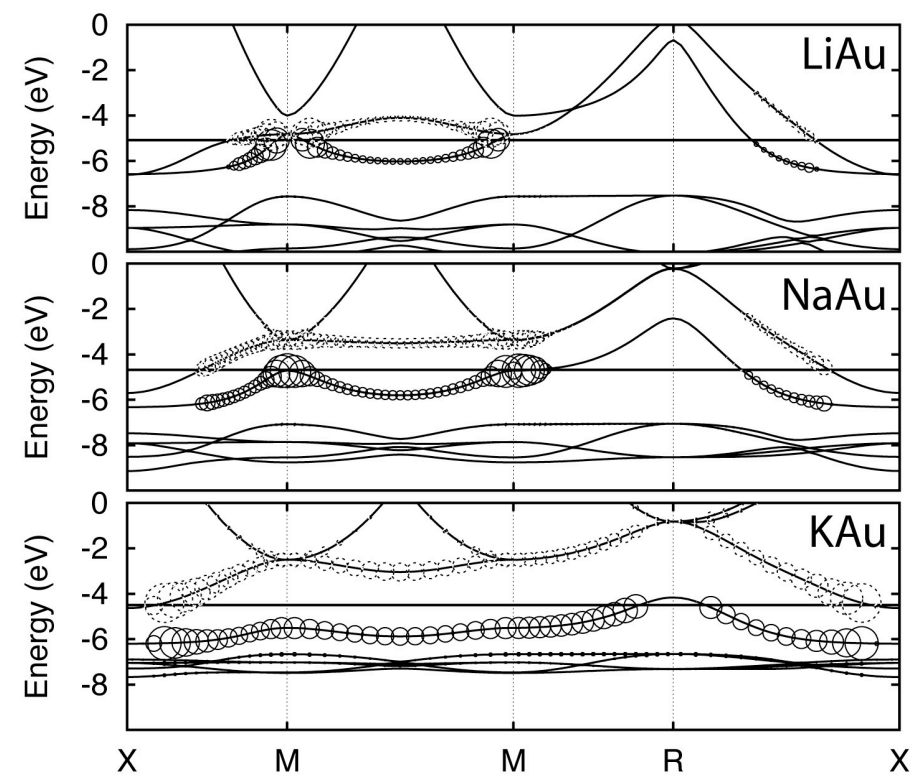

Figure 8. (Colour online) Band diagram for Alkali Au compounds. Included is the sum of transitions from a band below the Fermi energy (solid circles) to all bands above, and the sum of transitions to a band above the fermi energy (broken circles) from all bands below. The line M-M intersects X-R, see figure 8 .

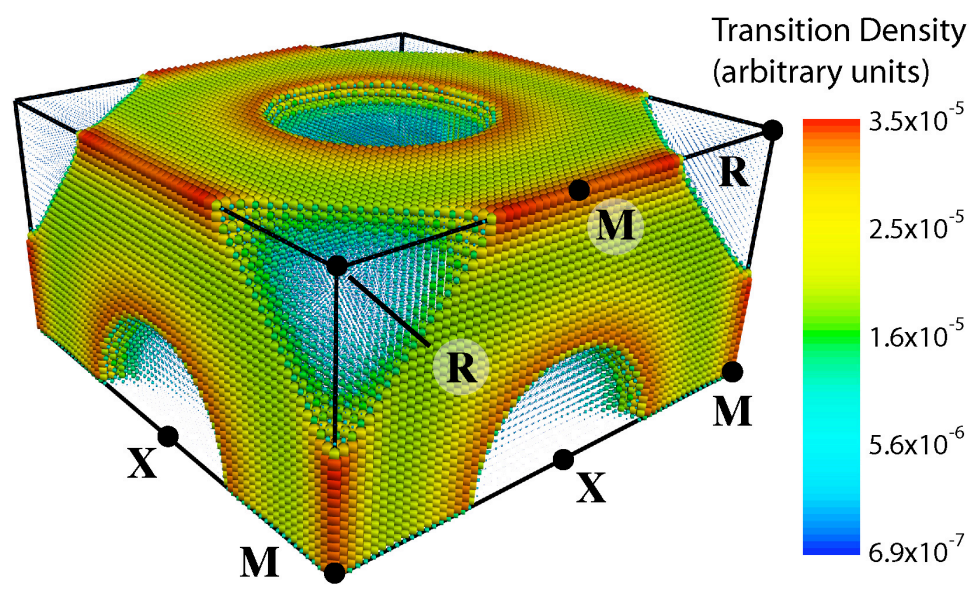

Figure 9. KAg: The sum of the transitions from all bands below the Fermi energy to all bands above the Fermi energy for each $\mathrm{k}$-point in the upper half $\left(+\mathrm{k}_{\mathrm{z}}\right)$ of the Brillouin zone.

\section{Conclusion}

In this paper we have employed Density Functional Theory to calculate the optical properties of a wide range of intermetallic compounds. The aim of this work is to systematically investigate how the optical properties vary with compound composition and identify trends useful to engineering compounds with optimal properties for plasmonic applications. We have concentrated our search to compounds composed of those elements which, on their own, have reasonable optical properties, that is the Alkali, Noble and Group III metals.

The Alkali Noble metals have by far the most potential with KAu having a band edge at almost the same energy as the plasma frequency. This fact, coupled with a low plasma frequency promises high quality plasmon resonances down to very low energies, most importantly, across the telecommunications wavelength $(\sim 1500 \mathrm{~nm})$. Both $\mathrm{NaAu}$ and $\mathrm{KAg}$ also have good band edge to plasma frequency ratios, resulting in the possibility of high quality plasmon resonances into the IR as well.

Alloying of Group III metals seems to substantially increase the low energy interband component of the permittivity way beyond that of elemental Aluminium, Gallium and Indium. The imaginary 
permittivity of Aluminium reaches only 46 at $1.5 \mathrm{eV}$ and this includes the Drude damping term. $\mathrm{AgAl}_{2}$, $\mathrm{AuAl}_{2}$ and most of the Alkali Noble Al compounds have imaginary permittivity in excess of 50 even without any Drude contribution. However, the plasma frequency of the Alkali-Noble-Group III compounds can be tuned between $2.84 \mathrm{eV}$ for $\mathrm{K}_{2} \mathrm{AuAl}$ to $8.62 \mathrm{eV}$ for $\mathrm{NaAu}_{2} \mathrm{Al}$. Although the quality of such resonances is debatable, this does allow for Drude like sphere resonances $\left(\omega=\omega_{p} / \sqrt{3}\right)$ [41] across the entire visible spectrum $(756 \mathrm{~nm}(1.64 \mathrm{eV})$ to $249 \mathrm{~nm}(4.98 \mathrm{eV}))$. There are select compounds in this series which show promise, in particular $\mathrm{Li}_{2} \mathrm{AgAl}$ has a small range of energies for which $\varepsilon^{\prime \prime}<1$ and $\mathrm{KAg}_{2} \mathrm{Al}$ has $\varepsilon^{\prime \prime}<1.3$.

\section{Acknowledgements}

This work was supported by the Australian Research Council, and the University of Technology, Sydney. Computing resources were provided by the Australian Centre for Advanced Computing and Communication (ac3) in New South Wales and the National Facility at the Australian Partnership for Advanced Computing (APAC). The authors acknowledge Prof M B Cortie for his extensive knowledge of intermetallics and for guiding us to the particular compounds in this study.

\section{References.}

[1] D. Pissuwan, S. M. Valenzuela, M. C. Killingsworth, X. D. Xu, and M. B. Cortie, Journal of Nanoparticle Research 9, 1109 (2007).

[2] D. Pissuwan, S. M. Valenzuela, C. M. Miller, and M. B. Cortie, Nano Lett. 7, 3808 (2007).

[3] L. R. Hirsch, R. J. Stafford, J. A. Bankson, S. R. Sershen, B. Rivera, R. E. Price, J. D. Hazle, N. J. Halas, and J. L. West, Proceedings of the National Academy of Sciences of the United States of America 100, 13549 (2003).

[4] S. Kawata, A. Ono, and P. Verma, Nat Photon advanced online publication (2008).

[5] N. Engheta, A. Salandrino, and A. Alu, Physical Review Letters 95, 095504 (2005).

[6] N. Engheta, Science 317, 1698 (2007).

[7] V. A. Fedotov, M. Rose, S. L. Prosvirnin, N. Papasimakis, and N. I. Zheludev, Physical Review Letters 99, 147401 (2007).

[8] J. B. Pendry, Physical Review Letters 85, 3966 (2000).

[9] J. B. Pendry, A. J. Holden, D. J. Robbins, and W. J. Stewart, Ieee Transactions on Microwave Theory and Techniques 47, 2075 (1999).

[10] J. B. Pendry, A. J. Holden, W. J. Stewart, and I. Youngs, Physical Review Letters 76, 4773 (1996).

[11] D. R. Smith, W. J. Padilla, D. C. Vier, S. C. Nemat-Nasser, and S. Schultz, Physical Review Letters 84, 4184 (2000).

[12] A. V. Kildishev, W. S. Cai, U. K. Chettiar, H. K. Yuan, A. K. Sarychev, V. P. Drachev, and V. M. Shalaev, Journal of the Optical Society of America B-Optical Physics 23, 423 (2006).

[13] U. K. Chettiar, A. V. Kildishev, T. A. Klar, and V. M. Shalaev, Optics Express 14, 7872 (2006).

[14] D. R. Smith, D. Schurig, M. Rosenbluth, S. Schultz, S. A. Ramakrishna, and J. B. Pendry, Applied Physics Letters 82, 1506 (2003).

[15] G. Dolling, M. Wegener, and S. Linden, Optics Letters 32, 551 (2007).

[16] S. Zhang, W. J. Fan, N. C. Panoiu, K. J. Malloy, R. M. Osgood, and S. R. J. Brueck, Physical Review Letters 95, 137404 (2005).

[17] J. Yao, Z. W. Liu, Y. M. Liu, Y. Wang, C. Sun, G. Bartal, A. M. Stacy, and X. Zhang, Science 321, 930 (2008).

[18] D. O. S. Melville and R. J. Blaikie, Optics Express 13, 2127 (2005).

[19] M. G. Blaber, M. D. Arnold, N. Harris, M. J. Ford, and M. B. Cortie, Physica BCondensed Matter 394, 184 (2007). 
[20] A. B. Scott, W. A. Smith, and M. A. Thompson, J. Phys. Chem. 57, 757 (1953).

[21] A. E. Hughes and S. C. Jain, Advances in Physics 28, 717 (1979).

[22] M. Zwilling, P. C. Schmidt, and A. Weiss, Applied Physics A: Materials Science \& Processing 16, 255 (1978).

[23] J. Y. Rhee, B. N. Harmon, and D. W. Lynch, Physical Review B 55, 4124 (1997).

[24] R. S. Rao, R. Prasad, and A. Bansil, Physical Review B 28, 5762 (1983).

[25] W. E. Spicer, A. H. Sommer, and J. G. White, Physical Review 115, 57 (1959).

[26] C. Koenig, N. E. Christensen, and J. Kollar, Physical Review B 29, 6481 (1984).

[27] G. H. Grosch and K. J. Range, Journal of Alloys and Compounds 233, 30 (1996).

[28] P. Ordejón, E. Artacho, and J. M. Soler, Physical Review B 53, R10441 (1996).

[29] J. M. Soler, E. Artacho, J. D. Gale, A. Garcia, J. Junquera, P. Ordejon, and D. SanchezPortal, Journal of Physics: Condensed Matter, 2745 (2002).

[30] N. Troullier and J. L. Martins, Physical Review B 43, 1993 (1991).

[31] J. Junquera, O. Paz, D. Sanchez-Portal, and E. Artacho, Physical Review B 64 (2001).

[32] J. P. Perdew, K. Burke, and M. Ernzerhof, Physical Review Letters 77, 3865 (1996).

[33] H. J. Monkhorst and J. D. Pack, Physical Review B 13, 5188 (1976).

[34] A. J. Read and R. J. Needs, Physical Review B 44, 13071 (1991).

[35] C. Kittel, Introduction to Solid State Physics (John Wiley \& Sons, New York, 1996).

[36] L. S. Hsu, G. Y. Guo, J. D. Denlinger, and J. W. Allen, Journal of Physics and Chemistry of Solids 62, 1047 (2001).

[37] J. H. Weaver and H. P. R. Frederikse, Optical properties of selected elements 82 ed. (CRC Press, Boca Raton, FL, 2001).

[38] S. S. Vishnubhatla and J. P. Jan, Philosophical Magazine 16, 45 (1967).

[39] V. V. Pavlyuk, G. S. Dmytriv, I. V. Chumak, H. Ehrenberg, and H. Pauly, Journal of Solid State Chemistry 178, 3303 (2005).

[40] R. E. Alonso, S. Sharma, C. Ambrosch-Draxl, C. O. Rodriguez, and N. E. Christensen, Physical Review B (Condensed Matter and Materials Physics) 73, 064101 (2006).

[41] Disregarding the interband component of the real part of the permittivity 